\title{
Sequential testing of $n$-out-of- $n$ systems: Precedence theorems and exact methods
}

\author{
Salim Rostami ${ }^{1,2}$, Stefan Creemers ${ }^{1}$, Wenchao $\mathrm{Wei}^{3}$ and Roel Leus ${ }^{2 *}$ \\ ${ }^{1}$ IÉSEG School of Management (LEM-CNRS 9221), Lille, France \\ ${ }^{2}$ ORSTAT, KU Leuven, Leuven, Belgium \\ ${ }^{3}$ School of Economics and Management, Beijing Jiaotong University, Beijing, China
}

\begin{abstract}
The goal of sequential testing is to discover the state of a system by testing its components one by one. We consider $n$-out-of- $n$ systems, which function only if all $n$ components work. The testing continues until the system state (up or down) is identified. The tests have known execution costs and failure probabilities, and are subject to precedence constraints. The objective is to find a sequence of tests that minimizes the total expected cost of the diagnosis. We show how to strengthen the precedence graph without losing all optimal solutions. We examine different formulations for the problem, and propose a dynamic-programming (DP) and a branch-and-price algorithm. Our computational results show that our DP noticeably outperforms the state of the art. Using a novel memory management technique, it significantly increases the size of the instances that can be solved to optimality within given limits on runtime and memory.
\end{abstract}

Keywords: sequential testing, $n$-out-of- $n$ systems, precedence theorems, dynamic programming, branchand-price.

History: Submitted April 2018. Revision submitted September 2018. Accepted October 2018.

\section{Introduction and related work}

A multi-component machine, e.g., a missile radar set, that is inactive except during a state of military alert, undergoes periodic checkups. The state of the machine (working or failing) depends on the state of its components. The components need to be tested in a specific order to detect the state of the machine with minimum cost or time. With these challenges in mind for preventive and corrective maintenance, the importance of sequential testing was highlighted by the U.S. Air Force more than sixty years ago [22]. The goal of sequential testing is to discover the state of a system by testing its components one by one. The testing continues until the system state is identified. Each test has a cost and a success probability. The objective is to find a sequence of tests that minimizes the total expected cost of the diagnosis.

\footnotetext{
${ }^{*}$ Corresponding author. E-mail: roel.leus@kuleuven.be, tel.: +32 16326967.
} 
As equipment becomes more complex and expensive, the number of required tests and the inspection costs increase, and consequently, the sequential diagnosis becomes more costly. From another perspective, as automated robotic machines replace human mechanics and quality control operators, it becomes vital to be able to prescribe a desirable sequence of inspections for machines [22]. In many practical applications, including rapid diagnosis in time-critical situations, e.g., toxic chemical identification [2], R\&D project scheduling [11, 13], quality control of manufactured products [17], container inspection operations at borders [25], and so on, the underlying problem incorporates sequential testing. Different tests may require different resources (e.g., manpower, electricity, etc.), or may entail other costs (e.g., pain to a patient). Therefore, examiners may try to postpone the more costly tests. Moreover, the tests are subject to precedence constraints. In pharmaceutical drug testing, for instance, animal (preclinical) tests precede human (clinical) tests [1].

An $n$-out-of- $n$ (also $n: n$ or serial) system is up if all its $n$ components are functioning. Hence, its testing terminates when the first failure is revealed. In a 1:n (parallel) system, on the other hand, a single working component guarantees the functionality of the whole system. From an optimization perspective, serial and parallel systems are equivalent: an optimal solution to a $1: n$ system with success probabilities $p_{i}$ is also optimal to an $n: n$ system with success probabilites $1-p_{i}$ [36]. A $k: n$ system is up if at least $k$ tests are successful, and is down if $(n-k+1)$ tests fail. Sequential testing of $k: n$ systems is studied by Wei et al. [39]. The state of a modular system, where each module represents a multi-part component, is up if all its modules are functional. A module is working if at least one of its parts functions. The modules and the tests within each module are precedence-related. Coolen et al. [9] and Creemers et al. [11] consider modular systems in project scheduling.

Various exact approaches have been proposed for special cases of sequential testing. It is shown that serial and parallel systems without precedence constraints are polynomially solvable $[4,6,21,22,27]$. A polynomial algorithm also exists for $k: n$ systems with arbitrary $k$, and without precedence constraints $[3,5,7,32,33]$. Serial systems are polynomially solvable when the precedence graph is a forest [20] or series-parallel [28]. An optimal diagnosis procedure is provided in [8] for $k: n$ systems with parallel-chain precedence constraints. Wagner and Davis [38] propose an integer-linear formulation for a single-item discrete sequential search problem, which is in fact a serial system known to be down. For an extensive literature review on sequential testing we refer to Ünlüyurt [36]. More recently, Daldal et al. [12] have offered approximation algorithms for sequential batch-testing of serial systems.

The subject of this article is sequential testing of $n: n$ systems with a general precedence graph, which is known to be strongly NP-hard [24]. To the best of our knowledge, no 
research has been specifically devoted to developing exact algorithms for this problem. The main contributions of this work are the following. (1) We show how to strengthen the precedence graph in a pre-processing step. (2) We develop a linear formulation for the problem obtained via Dantzig - Wolfe decomposition [26] of a compact nonlinear formulation. (3) A dynamic-programming (DP) and a branch-and-price (B\&P) algorithm are devised as solution methods. The computational results indicate that the DP procedure is faster than $\mathrm{B} \& \mathrm{P}$. Compared to the existing algorithms, our method significantly increases (by up to $100 \%$ ) the size of the instances that can be solved to optimality within given limits on runtime and memory. (4) In the process, we show that a relaxation of the pricing problem of our B\&P can be solved in polynomial time. We use this result to solve the pricing problem more efficiently.

Below, we first formally define the problem in Section 2. Section 3 is devoted to three precedence theorems for strengthening the precedence graph. We propose a DP and a B\&P algorithm in Sections 4 and 5, respectively. Computational results are provided in Section 6. We conclude in Section 7.

\section{Problem statement}

We consider a set $N$ of $n$ tests (or inspections) with pre-specified testing $\operatorname{costs} c_{i} \geq 0$, $i \in N$, and success probabilities $p_{i} \in[0,1]$. The set $E$ is a partial order on $N$, representing the precedence constraints. The precedence network of an instance of the problem can be visualized using an acyclic directed graph $G(N, E)$, where the nodes represent the tests, and there is an arc from node $i$ to $j$ iff $(i, j) \in E$. A test can be performed only if all its predecessors are done, where $j \in N$ is said to be a predecessor of $i \in N$ if $(j, i) \in E$. In a sequential diagnosis, tests are performed one at a time until the correct state of the system is identified. The system is functioning when all the $n$ tests are successful, and it is known to be down when the first failure is revealed.

A solution to the sequential testing problem is a decision policy that decides which test should be started next, given a history of previously performed tests and their results. For serial and parallel systems, there is a full order (sequence) of tests that is optimal [39]. This does not necessarily hold for $k: n$ systems. A full order is feasible iff it extends $E$. The objective is to find one sequence (list) $L=\left(l_{1}, l_{2}, \ldots, l_{n}\right)$ of the tests that minimizes the total expected cost of the diagnosis $\sum_{j=1}^{n}\left(\prod_{i=1}^{j-1} p_{l_{i}}\right) c_{l_{j}}$, where $\prod_{i} p_{i}$ over an empty set equals 1 .

Example: Consider the instance depicted in Figure 1 with the set $N$ of tests $\{1,2,3,4\}$, 


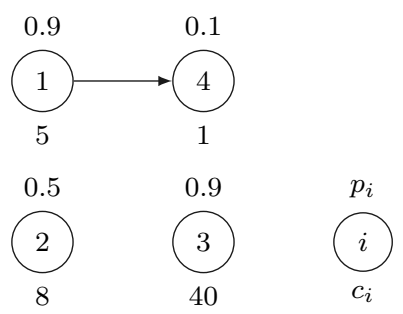

Figure 1: A problem instance

and the set of precedence constraints $E=\{(1,4)\}$. The sequence $L^{1}=(1,2,3,4)$ is a feasible solution with objective value $1 \times 5+0.9 \times 8+0.45 \times 40+0.405 \times 1=30.605$, while $L^{*}=(1,4,2,3)$ is an optimal sequence with objective value 8.42 .

Following Potts [30], we describe a compact formulation (CF) based on "linear ordering" variables $x_{i j}, i, j \in N$, where $x_{i j}=1$ if test $i$ precedes test $j$, and $x_{i j}=0$ otherwise.

$$
\begin{array}{lll}
\min & \sum_{j=1}^{n} c_{j} \prod_{i=1}^{n}\left[\left(p_{i}-1\right) x_{i j}+1\right] & \\
\text { s.t. } & x_{i j}+x_{j i}=1 & \\
& x_{j i}+x_{i k}-x_{j k} \leq 1 & \forall\{i, j\} \subset N \\
& x_{i j}=1 & \forall\{i, j, k\} \subset N \\
& x_{i j} \in\{0,1\} & \forall i, j) \in E
\end{array}
$$

Constraint (2.2) imposes anti-symmetry. Wagner and Davis [38] show that Constraint (2.3) is sufficient to enforce the transitivity property, and to eliminate cycles of any size. Constraint (2.4) enforces the precedence constraints in $E$.

Although correct, formulation CF is not practical due to its nonlinear objective. Following, we employ Dantzig-Wolfe decomposition (DW) [26] to linearize CF. DW as applied to integer programs entails a reformulation to express the integral solutions of a bounded convex polyhedron as integer convex combination of a finite set of points. The reformulation gives rise to another integer problem that typically has a tighter linear programming relaxation because it amounts to implicitly describing the convex hull of the integer polyhedron [37].

For any $i \in N$, let $\Gamma_{i}=\left\{x_{i}^{m} \in\{0,1\}^{n} \mid x_{j i}^{m}=1, \forall(j, i) \in E\right\}$ be the set of $\rho_{i}$ points $x_{i}^{m}=\left\{x_{1 i}^{m}, x_{2 i}^{m}, \ldots, x_{n i}^{m}\right\}$ that satisfy (2.4) and (2.5). Every $x_{i}^{m}$ corresponds to a set $C_{i}^{m} \subset N$ of candidate predecessors of test $i$, where $j \in C_{i}^{m}$ iff $x_{j i}^{m}=1$. Intuitively, $C_{i}^{m}$ is feasible iff for any $(j, i) \in E: j \in C_{i}^{m}$, for any $(i, k) \in E: k \notin C_{i}^{m}$, and $i \notin C_{i}^{m}$. We define $P_{i}^{m}=\prod_{j \in C_{i}^{m}} p_{j}$ as the joint success probability of $C_{i}^{m}$. 
Example (continued): Considering the previous example, for test 4, there are four different subsets, namely $C_{4}^{1}=\{1\}, C_{4}^{2}=\{1,2\}, C_{4}^{3}=\{1,3\}$, and $C_{4}^{4}=\{1,2,3\}$. The corresponding joint probabilities are $P_{4}^{1}=0.9, P_{4}^{2}=0.45, P_{4}^{3}=0.81$, and $P_{4}^{4}=0.41$. We observe that $C_{i}^{m}=\emptyset$ can also occur; for test 1 , for instance, we have $C_{1}^{1}=\emptyset$ with $P_{1}^{1}=1$.

Constraints (2.4) and (2.5) can be dropped from CF if we restrict the solution space to the sets $\Gamma_{i}$, i.e., if we impose

$$
\forall i \in N: x_{i}=\sum_{m=1}^{\rho_{i}} z_{i}^{m} x_{i}^{m}, \text { with } \sum_{m=1}^{\rho_{i}} z_{i}^{m}=1 \text { and } z_{i}^{m} \in\{0,1\}
$$

where the decision variable $z_{i}^{m}$ is 1 if the tests in $C_{i}^{m}$ precede $i$, and 0 otherwise. Using this transformation, and by replacing $\prod_{i=1}^{n}\left[\left(p_{i}-1\right) x_{j i}^{m}+1\right]$ by $P_{i}^{m}$ in the thus-obtained objective function, we reach the following integer linear formulation (ILF).

$$
\begin{aligned}
& (\mathrm{ILF}) \quad \min \sum_{i=1}^{n} c_{i} \sum_{m=1}^{\rho_{i}} P_{i}^{m} z_{i}^{m} \\
& \text { s.t. } \sum_{m=1}^{\rho_{j}} z_{j}^{m} x_{i j}^{m}+\sum_{m=1}^{\rho_{i}} z_{i}^{m} x_{j i}^{m}=1 \quad \forall\{i, j\} \subset N \\
& \sum_{m=1}^{\rho_{j}} z_{i}^{m} x_{j i}^{m}+\sum_{m=1}^{\rho_{k}} z_{k}^{m} x_{i k}^{m}-\sum_{m=1}^{\rho_{k}} z_{k}^{m} x_{j k}^{m} \leq 1 \quad \forall\{i, j, k\} \in N \\
& \sum_{m=1}^{\rho_{i}} z_{i}^{m}=1 \quad \forall i \in N \\
& z_{i}^{m} \in\{0,1\} \quad \forall i \in N, m \in\left\{1, \ldots, \rho_{i}\right\}
\end{aligned}
$$

The linear objective function of ILF comes at the cost of a high number of variables $z_{i}^{m}$ (exponential in $n$ ). To overcome this difficulty, in Section 5 , we propose an exact algorithm based on column generation for solving ILF.

\section{Precedence theorems}

A precedence constraint $(i, j) \in N \times N$ is said to be dominant iff there is at least one optimal solution with $i$ before $j$. Given an instance $G(N, E)$, let $D$ be the set of all dominant constraints. Precedence theorems may help us identify some of the constraints in $D$. Note that since every feasible solution satisfies the constraints in $E$, we have $E \subseteq D$. 


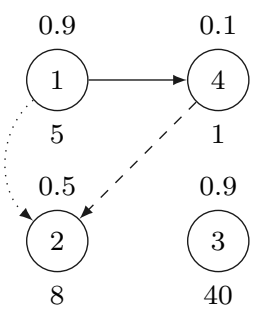

(a)

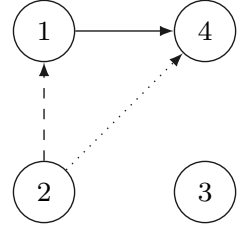

(b)

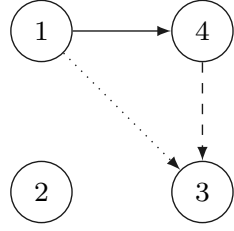

(c)

Figure 2: Possible extensions of the network in Figure 1

We know that when $E=\emptyset$, a sequence $L=\left(l_{1}, l_{2}, \ldots, l_{n}\right)$ is optimal [36] if

$$
\frac{c_{l_{1}}}{1-p_{l_{1}}} \leq \frac{c_{l_{2}}}{1-p_{l_{2}}} \leq \cdots \leq \frac{c_{l_{n}}}{1-p_{l_{n}}}
$$

The condition is intuitive: for a series system, it is advantageous to perform tests with low cost and high failure probability first. If $c_{i} /\left(1-p_{i}\right) \leq c_{j} /\left(1-p_{j}\right)$, then if $j$ precedes $i$ in a given sequence, we can swap the two tests, and the thus-obtained sequence cannot have a higher objective value than the initial sequence. We say the second sequence dominates the original.

Example (continued): For the previous example, we have $\frac{c_{1}}{1-p_{1}}=50, \frac{c_{2}}{1-p_{2}}=16, \frac{c_{3}}{1-p_{3}}=$ 400, and $\frac{c_{4}}{1-p_{4}}=1.11$. Since $\frac{c_{4}}{1-p_{4}}<\frac{c_{2}}{1-p_{2}}<\frac{c_{1}}{1-p_{1}}<\frac{c_{3}}{1-p_{3}}$, if we ignore the precedence constraints in $E$, then the sequence $(4,2,1,3)$ is optimal, with associated expected cost 3.85 . This sequence violates the precedence constraint $(1,4) \in E$, however. In Figure 2a, although $\frac{c_{4}}{1-p_{4}}<\frac{c_{2}}{1-p_{2}}$, since $(1,4) \in E$ (the solid edge), addition of the constraint $(4,2)$ (the dashed edge) implies the transitive constraint $(1,2)$ (the dotted edge), while $\frac{c_{2}}{1-p_{2}}<\frac{c_{1}}{1-p_{1}}$. Moreover, Figure $2 \mathrm{~b}$ shows that although $\frac{c_{2}}{1-p_{2}}<\frac{c_{1}}{1-p_{1}}$, adding the constraint $(2,1)$ also gives rise to the transitive constraint $(2,4)$, whereas $\frac{c_{4}}{1-p_{4}}<\frac{c_{2}}{1-p_{2}}$. Therefore, neither $(4,2)$ nor $(2,1)$ can be confirmed to be dominant. For $(4,3)$ in Figure 2c, on the other hand, we have $\frac{c_{4}}{1-p_{4}}<\frac{c_{3}}{1-p_{3}}$ and $\frac{c_{1}}{1-p_{1}}<\frac{c_{3}}{1-p_{3}}$. Consequently, the pair $(4,3)$ and the implied transitive edge $(1,3)$ are both dominant.

The previous example suggest an immediate extension of this rule for general $E$ : if for an activity pair $(i, j)$ we have $\frac{c_{i}}{1-p_{i}}<\frac{c_{j}}{1-p_{j}}$, and each of its transitive constraints from $E$ also satisfies the rule, then $(i, j)$ is dominant. Let $B_{i}=\{j \in N \mid(j, i) \in E\}$ and $A_{i}=\{j \in N \mid(i, j) \in E\}$ be the sets of tests that according to $E$ should be done before and after test $i$, respectively. For any $\{i, j\} \subset N$ with $(i, j),(j, i) \notin E$, we prove the following three theorems. Remark that for such a pair, $G(N, E \cup\{(i, j)\})$ is acyclic. 


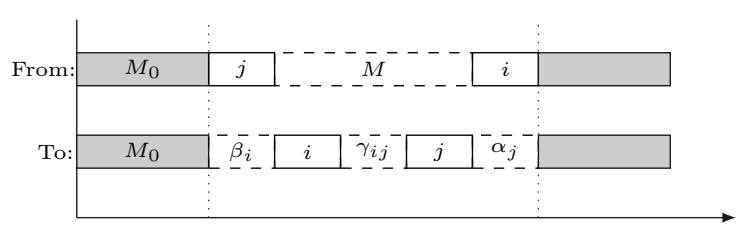

(a) Swap

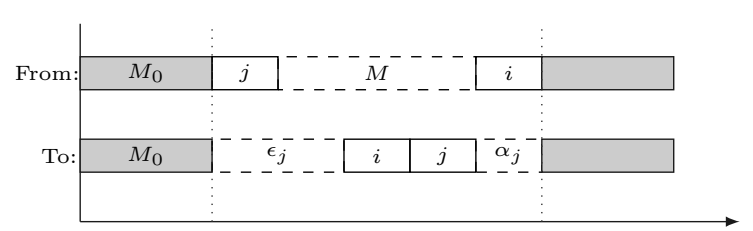

(b) Insert-after

Figure 3: Swap and insert-after strategies when $E \neq \emptyset$

Theorem 1. If for any $k \in\{i\} \cup B_{i} \backslash B_{j}$ and $q \in\{j\} \cup A_{j} \backslash A_{i}$ we have $c_{k} /\left(1-p_{k}\right) \leq c_{q} /\left(1-p_{q}\right)$, then $(i, j) \in D$.

Proof: We need to show that not only $(i, j)$ but also the associated transitive constraints are dominant. Consider the set $\left\{(k, q) \mid k \in B_{i}, q \in A_{j}\right\}$ of the pairs that are related to $(i, j)$. For general $E$, and for any $k \in B_{i} \cap B_{j}$ and $q \in A_{i} \cap A_{j}$, the pair $(k, q) \in E$, and consequently, is respected by every feasible solution. Therefore, we only need to check the condition $c_{k} /\left(1-p_{k}\right) \leq c_{q} /\left(1-p_{q}\right)$ for any $k \in\{i\} \cup B_{i} \backslash B_{j}$ and $q \in\{j\} \cup A_{j} \backslash A_{i}$. If the condition holds for every such pair, then the pair $(i, j)$ and its corresponding transitive pairs are dominant.

In the previous example, we saw that although $\frac{c_{4}}{1-p_{4}}<\frac{c_{2}}{1-p_{2}}$, constraint $(4,2)$ could not be identified as dominant because it also implies $(1,2)$, and $\frac{c_{2}}{1-p_{2}}<\frac{c_{1}}{1-p_{1}}$. Yet, it may be that the ratio $\frac{c_{4}}{1-p_{4}}$ is low enough that despite $\frac{c_{2}}{1-p_{2}}<\frac{c_{1}}{1-p_{1}},(4,2)$ is still dominant. To derive precedence theorems that can identify such cases we use the so-called "swap" and "insert-after" strategies $[23,31]$ to obtain two more precedence theorems. Given a feasible sequence with $j$ before $i$, and $(j, i) \notin E$, swapping the two jobs, or inserting $j$ after $i$ (together with the corresponding transitive changes), might lead to an improvement in the objective. The two strategies aim to find closed-form conditions to identify circumstances where such improvements are guaranteed. An illustration of these strategies for general $E$ is provided in Figure 3, where $\beta_{i}=\left(M \cap B_{i}\right), \alpha_{j}=\left(M \cap A_{j}\right), \gamma_{i j}=M \backslash\left(\alpha_{j} \cup \beta_{i}\right)$ and $\epsilon_{j}=M \backslash \alpha_{j}$, with $M$ the set of intermediate tests between $i$ and $j$. "From" represents any feasible sequence, and "To" is the resulting sequence after swapping $j$ and $i$, or inserting $j$ after $i$. Following the Swap strategy, a sufficient condition for the dominance of $(i, j)$ has the structure

$$
\mathrm{LB}(\mathrm{OI}(j)) \geq \mathrm{UB}(\mathrm{OD}(i))+\mathrm{UB}\left(\mathrm{OD}\left(\gamma_{i j}\right)\right)+\mathrm{UB}\left(\mathrm{OD}\left(\beta_{i}\right)\right)
$$

where $\operatorname{LB}(\cdot)$ and $\operatorname{UB}(\cdot)$ are lower and upper bound functions. For any $X \subseteq N$, we define $\mathrm{OI}(X)=\sum_{l \in X} \max \left\{0, c_{l} \prod_{k \prec_{\mathrm{T} l} l} p_{k}-c_{l} \prod_{k \prec_{\mathrm{From}} l} p_{k}\right\}$ as the objective improvement of the tests in $X$ after the changes, and $\operatorname{OD}(X)=\sum_{l \in X} \max \left\{0, c_{l} \prod_{k \prec \text { From } l} p_{k}-c_{l} \prod_{k \prec \text { To }} p_{k}\right\}$ as 
their objective degradation, where $i \prec_{L} j$ indicates that $i$ precedes $j$ in $L$. For simplicity of notation, we use $\mathrm{OI}(k)$ instead of $\mathrm{OI}(\{k\})$ for all singleton sets $X=\{k\}$, with $k \in N$. Note that $\mathrm{UB}\left(\mathrm{OD}\left(\alpha_{i}\right)\right)$ and $\mathrm{LB}\left(\mathrm{OI}\left(\alpha_{i}\right)\right)$ are set to 0 . A variant of Condition (3.12) can be used for the insert-after strategy by replacing $\mathrm{UB}\left(\mathrm{OD}\left(\gamma_{i j}\right)\right)+\mathrm{UB}\left(\mathrm{OD}\left(\beta_{i}\right)\right)$ by $\mathrm{UB}\left(\mathrm{OD}\left(\epsilon_{j}\right)\right)$.

If a pair $(i, j)$ of tests satisfies Condition (3.12) for every feasible $M$, and if $G(N, E \cup$ $\{(i, j)\})$ is acyclic, then if $j$ precedes $i$ in a given schedule, we can exchange the two tests without increasing the cost. Thus, for a set of test pairs $\{(i, j),(k, q), \ldots\}$ that each satisfy one or both of the swap and insert-after dominance conditions, any optimal schedule that is not compatible with one or more of these pairs cannot be deteriorated by making as many interchanges as necessary to obtain an optimal schedule that respects all the pairs. Let $S \subseteq D$ with $S \supseteq E$ be the set of dominant precedence constraints that are previously identified. Starting from $S=E$, we iteratively identify dominant precedence constraints, which are then added to $S$. The iterative extension of $S$ stops only when we can no longer identify new dominant constraints. We conclude:

Proposition 1. There is at least one optimal solution that respects all the precedence constraints in $S$ iff the graph $G(N, S)$ is acyclic.

Similar to $B_{i}$ and $A_{i}$, we define $B_{i}^{S}$ and $A_{i}^{S}$ based on $S$ instead of $E$. Moreover, for any $X \subseteq N$, we define $P(X)=\prod_{i \in X} p_{i}$, and $\mathrm{CF}[X]$ is the subproblem, where $N$ and $E$ in $\mathrm{CF}$ are replaced by $X$ and $\{(i, j) \in E \mid i, j \in X\}$, respectively. Finally, $\lambda\left(B_{i}^{S}\right), \theta\left(\gamma_{i j}\right)$, and $\phi\left(\epsilon_{j}\right)$ are upper bounds to the subproblems $\mathrm{CF}\left[B_{i}^{S}\right], \max _{\gamma_{i j} \subseteq N \backslash\left(\{j\} \cup B_{j}^{S} \cup A_{j}^{S} \cup\{i\} \cup B_{i}^{S} \cup A_{i}^{S}\right)}\left\{\mathrm{CF}\left[\gamma_{i j}\right]\right\}$, and $\max _{\epsilon_{j} \supseteq B_{i}^{S} \backslash B_{j}^{S}, \epsilon_{j} \subseteq N \backslash\left(\{j\} \cup B_{j}^{S} \cup A_{j}^{S} \cup\{i\} \cup A_{i}^{S}\right)}\left\{\mathrm{CF}\left[\epsilon_{j}\right]\right\}$, respectively, where $\gamma_{i j}$ and $\epsilon_{j}$ are precedencefeasible subsets of tests.

Theorem 2. If

$$
\begin{aligned}
& c_{j}\left(1-p_{i}\right) P\left(N \backslash\left(\{j\} \cup A_{j}^{S} \cup\{i\} \cup A_{i}^{S}\right)\right) \geq \\
& \quad\left(c_{i} P\left(B_{j}^{S} \cup B_{i}^{S}\right)+P\left(B_{j}^{S}\right) \lambda\left(B_{i}^{S}\right)\right)\left(1-p_{j} P\left[N \backslash\left(\{j\} \cup B_{j}^{S} \cup\{i\} \cup B_{i}^{S} \cup A_{i}^{S}\right)\right]\right) \\
& \quad+P\left(B_{j}^{S} \cup B_{i}^{S}\right) \theta\left(\gamma_{i j}\right) \max \left\{0, p_{i}-p_{j} P\left(A_{j}^{S}\right)\right\},
\end{aligned}
$$

then $(i, j) \in D$.

Proof: For Condition (3.12) for the swap strategy we have

$$
\begin{aligned}
\mathrm{OI}(j) & =\max \left\{0, P\left(M_{0}\right) c_{j}-P\left(M_{0}\right) P\left(\beta_{i}\right) p_{i} P\left(\gamma_{i j}\right) c_{j}\right\} \\
& =P\left(M_{0}\right) c_{j}\left(1-p_{i} P\left(\beta_{i}\right) P\left(\gamma_{i j}\right)\right)
\end{aligned}
$$




$$
\geq c_{j}\left(1-p_{i}\right) P\left[N \backslash\left(\{j\} \cup A_{j}^{S} \cup\{i\} \cup A_{i}^{S}\right)\right],
$$

where $M_{0}$ is the set of tests that precede $j$ in From sequence in Figure 3a.

$$
\begin{aligned}
\mathrm{OD}(i) & =\max \left\{0, P\left(M_{0}\right) P\left(\beta_{i}\right) c_{i}-P\left(M_{0}\right) p_{j} P(M) c_{i}\right\} \\
& =c_{i} P\left(M_{0}\right) P\left(\beta_{i}\right)\left(1-p_{j} P\left(M \backslash \beta_{i}\right)\right) \\
& \leq c_{i} P\left(B_{j}^{S} \cup B_{i}^{S}\right)\left(1-p_{j} P\left[N \backslash\left(\{j\} \cup B_{j}^{S} \cup\{i\} \cup B_{i}^{S} \cup A_{i}^{S}\right)\right]\right) . \\
\mathrm{OD}\left(\beta_{i}\right) & \leq \max \left\{0, P\left(M_{0}\right) \lambda\left(\beta_{i}\right)-P\left(M_{0}\right) p_{j} P\left(M \backslash \beta_{i}\right) \lambda\left(\beta_{i}\right)\right\} \\
& =P\left(M_{0}\right) \lambda\left(\beta_{i}\right)\left(1-p_{j} P\left(M \backslash \beta_{i}\right)\right) \\
& \leq P\left(B_{j}^{S}\right) \lambda\left(B_{i}^{S}\right)\left(1-p_{j} P\left[N \backslash\left(\{j\} \cup B_{j}^{S} \cup\{i\} \cup B_{i}^{S} \cup A_{i}^{S}\right)\right]\right) . \\
\mathrm{OD}\left(\gamma_{i j}\right) & \leq \max \left\{0, P\left(M_{0}\right) P\left(\beta_{i}\right) p_{i} \theta\left(\gamma_{i j}\right)-P\left(M_{0}\right) p_{j} P\left(\alpha_{j}\right) P\left(\beta_{i}\right) \theta\left(\gamma_{i j}\right)\right\} \\
& =P\left(M_{0}\right) P\left(\beta_{i}\right) \theta\left(\gamma_{i j}\right) \max \left\{0, p_{i}-p_{j} P\left(\alpha_{j}\right)\right\} \\
& \leq P\left(B_{j}^{S} \cup B_{i}^{S}\right) \theta\left(\gamma_{i j}\right) \max \left\{0, p_{i}-p_{j} P\left(A_{j}^{S}\right)\right\} .
\end{aligned}
$$

By substituting the expressions above in (3.12), we have (3.13).

Theorem 3. If

$$
\begin{aligned}
& c_{j}\left(1-p_{i}\right) P\left(N \backslash\left(\{j\} \cup A_{j}^{S} \cup\{i\} \cup A_{i}^{S}\right)\right) \geq \\
& \left(c_{i} P\left(B_{j}^{S} \cup B_{i}^{S}\right)+P\left(B_{j}^{S}\right) \phi\left(\epsilon_{j}\right)\right)\left(1-p_{j} P\left(A_{j}^{S}\right)\right),
\end{aligned}
$$

then $(i, j) \in D$.

Proof: For Condition (3.12) for the insert-after strategy we have

$$
\begin{aligned}
\mathrm{OI}(j) & \geq c_{j}\left(1-p_{i}\right) P\left[N \backslash\left(\{j\} \cup A_{j}^{S} \cup\{i\} \cup A_{i}^{S}\right)\right] . \\
\mathrm{OD}(i) & =\max \left\{0, P\left(M_{0}\right) P\left(\epsilon_{j}\right) c_{i}-P\left(M_{0}\right) p_{j} P(M) c_{i}\right\} \\
& =c_{i} P\left(M_{0}\right) P\left(\epsilon_{j}\right)\left(1-p_{j} P\left(\alpha_{j}\right)\right) \\
& \leq c_{i} P\left(B_{j}^{S} \cup B_{i}^{S}\right)\left(1-p_{j} P\left(A_{j}^{S}\right)\right) . \\
\mathrm{OD}\left(\epsilon_{j}\right) & \leq \max \left\{0, P\left(M_{0}\right) \phi\left(M \backslash \alpha_{j}\right)-P\left(M_{0}\right) p_{j} P\left(\alpha_{j}\right) \phi\left(\epsilon_{j}\right)\right\} \\
& =P\left(M_{0}\right) \phi\left(\epsilon_{j}\right)\left(1-p_{j} P\left(\alpha_{j}\right)\right) \\
& \leq P\left(B_{j}^{S}\right) \phi\left(\epsilon_{j}\right)\left(1-p_{j} P\left(A_{j}^{S}\right)\right) .
\end{aligned}
$$

By substituting the expressions above in (3.12) we have (3.14). 
There is an obvious trade-off between the quality of the bounds $\lambda\left(B_{i}^{S}\right), \theta\left(\gamma_{i j}\right)$ and $\phi\left(\epsilon_{j}\right)$, and the time complexity of their computation. Here, we opt for obtaining these bounds in polynomial time. We obtain $\lambda\left(B_{i}^{S}\right)$ by ordering the tests in non-decreasing order of $c_{i} /\left(1-p_{i}\right)$ while respecting the precedence constraints. That is, starting from the first position of an empty list, we fill the $n$ positions, iteratively. At each of the $n$ decision points, we choose the eligible test with lowest $c_{i} /\left(1-p_{i}\right)$. For $\theta\left(\gamma_{i j}\right)$ and $\phi\left(\epsilon_{j}\right)$, one straightforward approach is to order the tests in non-increasing order of $c_{i} /\left(1-p_{i}\right)$, ignoring the precedence constraints.

Example (continued): For the previous example, we obtain $(2,1,4,3)$ for $\lambda(N)$ with the objective value 12.75. As an upper bound to $\max _{X \subseteq N}\{\mathrm{CF}[X]\}$ (as for $\theta$ and $\phi$ bounds), on the other hand, we obtain $(3,1,2,4)$ with the objective value 51.385. For this example, we can show that Theorems 1 and 3 identify $(1,3),(2,3)$, and $(4,3)$ as dominant, while Theorem 2 identifies $(4,2)$ and $(4,3)$. The identified constraints are respected by the optimal solution $L^{*}=(1,4,2,3)$. Note that Theorem 2 identifies $(4,2)$ as dominant, despite the fact that for its transitive constraint $(1,2)$, we have $\frac{c_{2}}{1-p_{2}}<\frac{c_{1}}{1-p_{1}}$.

In the remainder, we assume that the input precedence networks have been tightened using the precedence theorems, but we maintain the notation $E$ instead of $S$ for the set of precedence constraints. We also assume that the test indices form a topological order of $G(N, E)$.

\section{DP algorithm}

In this section, we propose a DP algorithm for solving the sequential testing problem for $n: n$ systems. In the remainder of the text, we will refer to this algorithm as DPn. The recursion of DPn (Section 4.1) is close to the recursion in [9, 39], but tuned specifically towards the $n: n$ problem. We use an improved version of the memory management technique in [31] (Section 4.2). This technique enables us to solve significantly larger instances (by up to $100 \%$ ) than before within given limits on runtime and memory.

\subsection{DP recursion}

Each state is defined as a set $Y \subseteq N$ of to-be-scheduled tests. Every state $Y$ represents a sequential testing subproblem, where the first $n-|Y|$ positions of the sequence are filled, and we decide the test that occupies the $(n-|Y|+1)^{\text {th }}$ position. The state space $\Phi$ contains all the feasible states. A state $Y$ is feasible if it respects the precedence constraints, i.e., $\forall i \in Y: A_{i} \subset Y$. Given a state $Y \in \Phi$, let $\xi(Y)=\left\{i \in Y \mid B_{i} \cap Y=\emptyset\right\}$ be the set of tests 


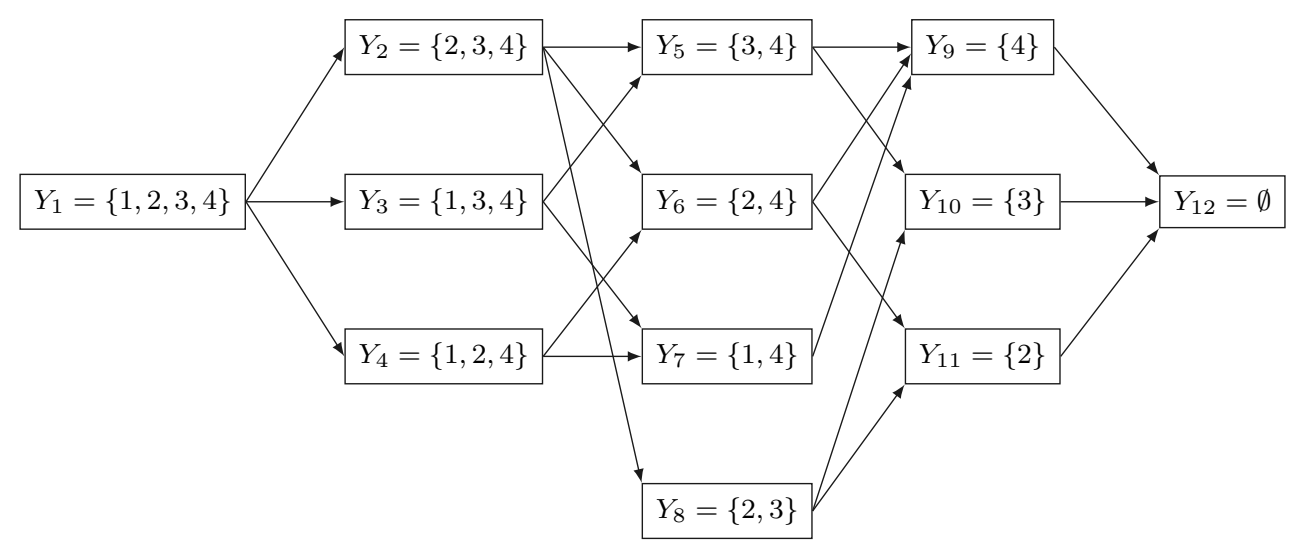

Figure 4: The state space of the example in Figure 1

that are eligible to be tested. Hence, $\xi(Y)$ contains all the possible decisions to be made in $Y$. Selecting test $i \in \xi(Y)$ leads to $Y \backslash\{i\}$ as the immediate succeeding state. The value function of any state $Y$ can be computed via the backward recursion

$$
G(Y)=\min _{i \in \xi(Y)}\left\{c_{i}+p_{i} G(Y \backslash\{i\})\right\}
$$

Starting from the unique final state $Y=\emptyset$ with $G(\emptyset)=0$, DPn iteratively calculates the objective value corresponding to the preceding states.

Example (continued): Figure 4 depicts the state space of the previous example. The objective value of $Y_{9}$ is $c_{4}+p_{4} \times G(\emptyset)=1$, while $G\left(Y_{2}\right)=\min \left\{c_{2}+p_{2} \times G\left(Y_{5}\right), c_{3}+p_{3} \times\right.$ $\left.G\left(Y_{6}\right), c_{4}+p_{4} \times G\left(Y_{8}\right)\right\}=3.8$. Finally, $G\left(Y_{1}\right)=8.42$ is the optimal objective value; the unique optimal solution is $L^{*}=(1,4,2,3)$.

\subsection{DP memory management}

In this section we elaborate an improved version of the memory management technique that is proposed in [31] for precedence-constrained single machine scheduling with weighted tardiness objective. The method partitions the state space into sets of states with equal cardinality, i.e., it divides $\Phi$ into sets $\Upsilon^{f}=\{Y \in \Phi:|Y|=f\}$, with $0 \leq f \leq n$. In Figure 4, each $\Upsilon^{f}$ corresponds to one "column" of states, e.g., $\Upsilon^{2}=\left\{Y_{5}, Y_{6}, Y_{7}, Y_{8}\right\}$. For a given $Y \in \Phi$, let $Q(Y)=\left\{i \in N \backslash Y \mid A_{i} \subseteq Y\right\}$ be the set of tests in $N \backslash Y$ with all successors in $Y$. Hence, for any $i \in Q(Y), Y \cup\{i\}$ is a feasible state. For a given $f(0<f \leq n)$ we have

$$
\Upsilon^{f}=\bigcup_{Y \in \Upsilon^{f-1}}\{Y \cup\{i\} \mid i \in Q(Y)\}
$$




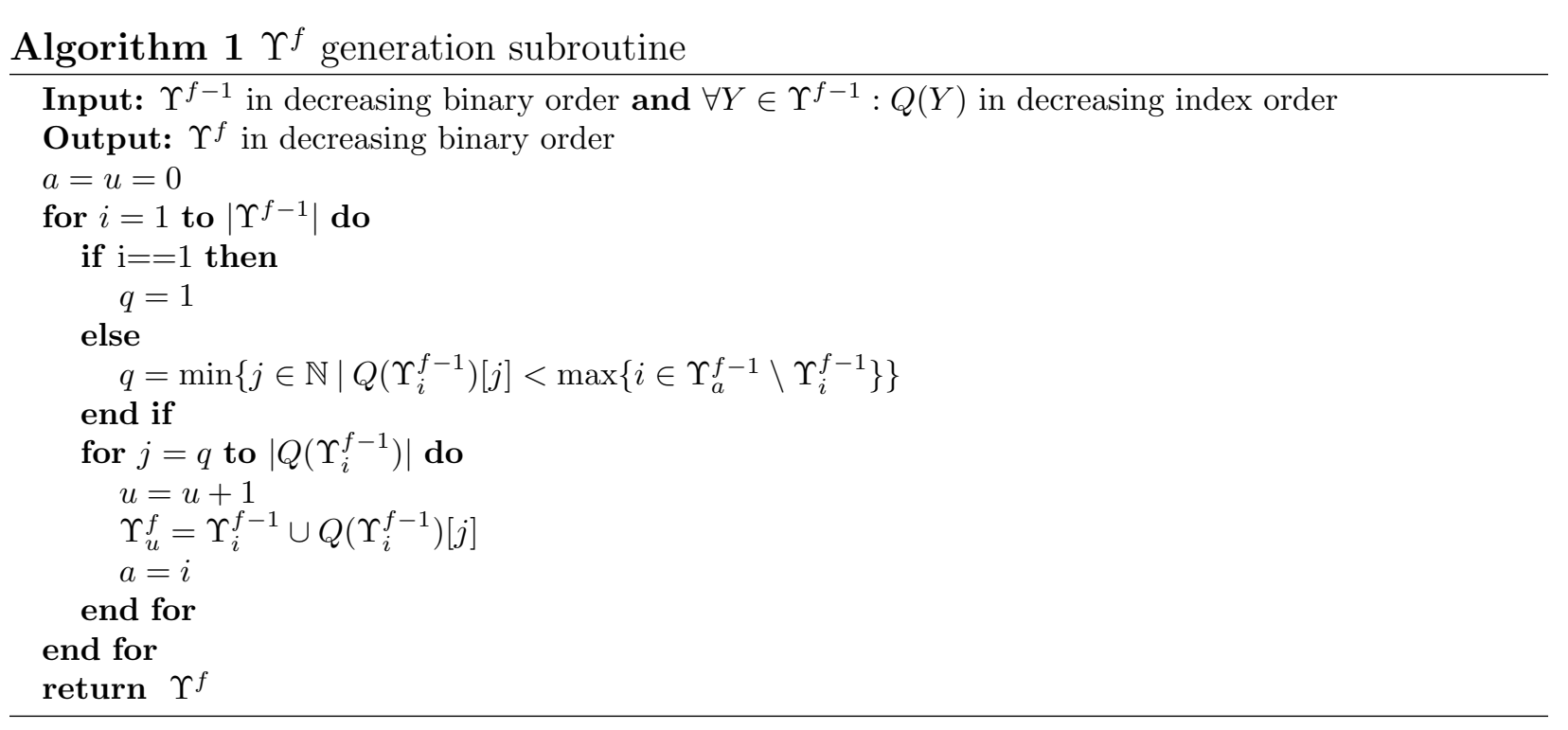

and $\Upsilon^{0}=\{\emptyset\}$. Therefore, the generation of $\Upsilon^{f}$ depends only on $\Upsilon^{f-1}$. Moreover, according to (4.15), for any state $Y \in \Upsilon^{f}$ the objective value $G(Y)$ is determined by the objective value of states in $\Upsilon^{f-1}$. We conclude that, once $\Upsilon^{f}$ is created, and the objective value of its members is computed, the set $\Upsilon^{f-1}$ can be discarded from memory. This means that at any time during the execution of DP, we need to store at most two sets of states $\left(\Upsilon^{f-1}\right.$ and $\left.\Upsilon^{f}\right)$.

One drawback of the method of Rostami et al. [31] is that for each newly generated state, it has to be checked if the state has been generated before. If a state already exists in the set of generated states, then we only need to update its objective value. In this article we propose an improved version of the technique that does not require the aforementioned checks, and is consequently faster.

Algorithm 1 elaborates a subroutine that receives $\Upsilon^{f-1}$, and generates $\Upsilon^{f}$, where $Q(Y)[i]$ is the $i^{\text {th }}$ element in $Q(Y), \Upsilon_{i}^{f}$ is the $i^{\text {th }}$ element of $\Upsilon^{f}$, and $u$ is a counter of the states in $\Upsilon^{f}$ that are previously generated. With each state $Y$, we associate $\tau(Y)=\sum_{i \in Y} 2^{i-1}$ as its binary representation. The elements of the resulting $\Upsilon^{f}$ are generated in decreasing order of their binary representation if the states in $\Upsilon^{f-1}$ are processed in decreasing binary order, and for each state $Y \in \Upsilon^{f-1}$, the tests in $Q(Y)$ are scanned in decreasing index order.

Example (continued): In Figure 4, and for $\Upsilon^{2}$, the states are in decreasing binary order: $\Upsilon_{1}^{2}=Y_{5}, \Upsilon_{2}^{2}=Y_{6}, \Upsilon_{3}^{2}=Y_{7}$, and $\Upsilon_{4}^{2}=Y_{8}$ with $\tau\left(Y_{5}\right)=12, \tau\left(Y_{6}\right)=10, \tau\left(Y_{7}\right)=9$, and $\tau\left(Y_{8}\right)=6$. The states that are generated from $Y_{5}$, are in decreasing binary order if we scan the tests in $Q\left(Y_{5}\right)=\{1,2\}$ in decreasing index order, meaning $Q\left(Y_{5}\right)[1]=2$ and $Q\left(Y_{5}\right)[2]=1$. In this case, $Y_{2}=Y_{5} \cup\{2\}$ with $\tau\left(Y_{2}\right)=14$, and $Y_{3}=Y_{5} \cup\{1\}$ with $\tau\left(Y_{3}\right)=13$. 
Suppose the states $\Upsilon_{u-1}^{f}$ and $\Upsilon_{u}^{f}$ are generated from the states $\Upsilon_{a}^{f-1}$ and $\Upsilon_{b}^{f-1}(a<b)$, respectively. We define $h=\max \left\{i \in \Upsilon_{a}^{f-1} \backslash \Upsilon_{b}^{f-1}\right\}$.

Theorem 4. In Algorithm 1, the state $\Upsilon_{b}^{f-1} \cup\{i\}$ with $i \in Q\left(\Upsilon_{b}^{f-1}\right)$ has already been generated iff $i \geq h$.

Proof: We define $g=\min \left\{i \in Y_{b}^{f-1}\right\}$. It can be shown that $h>g$. Assume that $\Upsilon_{u}^{f}$ is produced by adding $Q\left(\Upsilon_{b}^{f-1}\right)[q]$ to $\Upsilon_{b}^{f-1}$, and that $Q\left(\Upsilon_{b}^{f-1}\right)[q] \geq h$. Since the test indices form a topological order of $G(N, E)$, we know that $B_{g} \cap \Upsilon_{b}^{f-1}=\emptyset$ and $g \notin A_{Q\left(\Upsilon_{b}^{f-1}\right)[q]}$. Consequently, $Y^{*}=\Upsilon_{b}^{f-1} \backslash\{g\} \cup\left\{Q\left(\Upsilon_{b}^{f-1}\right)[q]\right\}$ is a feasible state with cardinality $f-1$. Since $\tau\left(Y^{*}\right)>\tau\left(\Upsilon_{b}^{f-1}\right)$, there exists $c \leq a$ such that the state $\Upsilon_{c}^{f-1}=Y^{*}$. Knowing that $Y^{\prime}=Y^{*} \cup\{g\}$, we conclude that $Y^{\prime}$ has already been generated. On the other hand, if $Q\left(\Upsilon_{b}^{f-1}\right)[q]<h$, then $\Upsilon_{u}^{f}<\Upsilon_{u-1}^{f}$, and since the elements in $\Upsilon^{f}$ are generated in decreasing binary order, we conclude that $\Upsilon_{u}^{f}$ has not been generated before.

Example (continued): For the states that are generated from $Y_{6}$, we have $h=\max \{i \in$ $\left.Y_{5} \backslash Y_{6}\right\}=3$ and $g=\min \left\{i \in Y_{6}\right\}=2$. Since $Q\left(Y_{6}\right)=\{1,3\}$, we conclude that the state $Y_{6} \cup\{3\}$ is already generated $\left(Y_{2}\right)$ from the state $Y_{6} \backslash\{2\} \cup\{3\}=Y_{5}$. The state $Y_{4}=Y_{6} \cup\{1\}$, on the other hand, is new.

Wei et al. [39] use uniformly directed cuts (UDCs) to structure the state space $\Phi$. Each UDC $u \subseteq N$ is an inclusion-maximal set of incomparable tests, i.e., for any $\{i, j\} \in u$ : $(i, j) \notin E \wedge(j, i) \notin E$. Let $U$ be the set of all UDCs. With each UDC $u \in U$, Wei et al. associate a set $\sigma(u) \subset \Phi$ of states in which one or more tests in $u$ are eligible to start, and the tests in $N \backslash u$ are all ineligible or finished. It is shown in [10] that the sets $\sigma(u)$ are mutually exclusive, and $\bigcup_{u \in U} \sigma(u)=\Phi$. Hence, $U$ is a valid partition of $\Phi$, and allows, at each time during the optimization, to store and process only a subset of $\Phi$, and to dismiss the states that will no longer be used. The number of UDCs is exponential in $n$, and they have to be enumerated prior to optimization. Our method, on the other hand, is not dependent on UDCs, and combines the generation of the states with the calculation of their objective value during optimization. In Section 6, we empirically show that our memory management technique not only reduces the computational effort required to generate and search the state space, but also reduces the number of states that are stored in memory simultaneously. In other words, it is not only faster but also more memory-efficient. 


\section{Column generation}

Column generation (CG) [26] is a technique for solving linear programs with a large number of variables. The algorithm consists of a master and a pricing problem. Here, the linear relaxation of ILF, denoted by LF, is the master problem. It is called restricted (RLF) when it includes only a subset of its variables (columns). RLF is solved iteratively, and at each iteration, the pricing problem determines whether a new variable exists that if added to the restricted master problem, will improve its objective value. If no such variable exists then an optimal solution to the current RLF is also optimal to LF. A heuristic algorithm (see Section 6.2) is used to obtain the initial columns of RLF.

\subsection{The pricing problem}

Let $\bar{\beta}_{i j}, \kappa_{i j k}$, and $\alpha_{i}$ be the dual variables attached to Constraints (2.7), (2.8), and (2.9), respectively. The reduced cost of the variable $z_{i}^{m}$ is then

$$
P_{i}^{m} c_{i}-\alpha_{i}-\sum_{j \in N \backslash\{i\}} \bar{\beta}_{i j} x_{j i}^{m}-\sum_{j \in N \backslash\{i\}} \sum_{k \in N \backslash\{i, j\}} \kappa_{i j k} x_{j i}^{m} .
$$

The pricing problem then boils down to the question $\exists i, m: P_{i}^{m} c_{i}-\alpha_{i}-\sum_{j \in C_{i}^{m}} \beta_{i j}<$ 0 ?, where $\beta_{i j}=\bar{\beta}_{i j}+\sum_{k \in N \backslash\{i, j\}} \kappa_{i j k}$. A new entering variable with negative reduced cost corresponds to a new set $C_{i}^{m}$. To find such variables, at each iteration, and for every $i \in N$, we solve the pricing problem

$$
\begin{array}{lll}
\min & F\left(N_{i}, \varsigma_{i}, \beta_{i}\right)=\varsigma_{i} \prod_{j \in N_{i}} p_{j}^{y_{j}}-\sum_{j \in N_{i}} \beta_{i j} y_{j} & \\
\text { s.t. } & y_{j} \geq y_{k} & \forall(j, k) \in E \\
& y_{j} \in\{0,1\} & \forall j \in N_{i}
\end{array}
$$

where $N_{i}=N \backslash\left(B_{i} \cup A_{i} \cup\{i\}\right)$ is the set of tests that are not precedence-related to $i$, and $\varsigma_{i}=c_{i} \prod_{j \in B_{i}} p_{j}$. The decision variable $y_{j}$ is equal to 1 if $j$ is selected, and 0 otherwise. For given arguments, the objective value of an optimal solution $\mathbf{y}^{*}$ is denoted by $F\left(\mathbf{y}^{*}\right)$ or $F^{*}$. If there exists $i \in N$ such that $F\left(N_{i}, \varsigma_{i}, \beta_{i} ; \mathbf{y}^{*}\right)<\alpha_{i}+\sum_{j \in B_{i}} \beta_{i j}$, then the subset $C_{i}^{m}=\left\{j \in N_{i} \mid y_{j}^{*}=1\right\} \cup B_{i}$ and its corresponding variable $z_{i}^{m}$ should enter RLF; otherwise, a current optimal solution to RLF is also optimal to LF. 


\subsubsection{A DP algorithm for solving PR}

In this section, we develop a DP algorithm for solving the pricing problem PR. Henceforth, we refer to this algorithm as DPp. The problem bears similarities with the precedenceconstrained knapsack problem. The main framework of DPp is also inspired by the DP algorithm of Samphaiboon and Yamada [34].

For the pricing problem $\operatorname{PR}\left[N_{i}, \varsigma_{i}, \beta_{i}\right]$, a DP state is defined by $(Y, Z)$, where $Y \subseteq N_{i}$ is a set of tests that may or may not be selected in the new set of predecessors of $i$, and $Z \subseteq\left(N_{i} \cup B_{i}\right) \backslash Y$ with $Z \supseteq B_{i}$ is the set of tests that are chosen to be in the set of predecessors of $i$. Each DP state $(Y, Z)$ represents a subproblem $\operatorname{PR}\left[Y, \varsigma, \beta_{i}\right]$, where $\varsigma=c_{i} \prod_{j \in Z} p_{j}$. Let $\Omega$ be the set of all feasible states. A state $(Y, Z)$ is called feasible if it respects the precedence constraints in $E$, i.e., for each $j \in Y: B_{j} \subset(Y \cup Z)$, and for each $j \in Z: B_{j} \subset Z$. A state $(Y, Z)$ is called singleton if $|Y|=1$. We define $\delta(Y)=\min \{j \in Y\}$ as the test with minimum index in $Y$. Assuming that the test indices form a topological order of $G(N, E)$, we have $B_{\delta(Y)} \cap Y=\emptyset$. Considering a DP state $(Y, Z)$, fixing $y_{\delta(Y)}=1$ leads to $(Y \backslash\{\delta(Y)\}, Z \cup\{\delta(Y)\})$, and the choice $y_{\delta(Y)}=0$ results in $\left(Y \backslash\left(\{\delta(Y)\} \cup A_{\delta(Y)}\right), Z\right)$. For a given state $(Y, Z)$, the value function $\bar{F}$ computes the minimum reduced cost via the following recursion.

$$
\begin{gathered}
\bar{F}^{*}(Y, Z)=\min \left\{\bar{F}^{*}(Y \backslash\{\delta(Y)\}, Z \cup\{\delta(Y)\})+R(Z, Y),\right. \\
\left.\bar{F}^{*}\left(Y \backslash\left(\{\delta(Y)\} \cup A_{\delta(Y)}\right), Z\right)\right\},
\end{gathered}
$$

where $R(Y, Z)=c_{i} \prod_{j \in Z} p_{j}\left(p_{\delta(Y)}-1\right)-\beta_{i, \delta(Y)}$ is the reward of selecting $\delta(Y)$, and $\bar{F}^{*}(\emptyset, Z)=$ 0 . The optimal decision variable for each state $(Y, Z)$ is denoted by $\mathbf{y}^{*}(Y, Z)$.

Example (continued): In the previous example, the pricing problem corresponding to test 3 is denoted by $\operatorname{PR}\left[N_{3}, \varsigma_{3}, \beta_{3}\right]$, where $N_{3}=\{1,2,4\}, B_{3}=\emptyset, c_{3}=40$ Let us illustrate the calculation with $\beta_{3}=\{-10,-20,0,-20\}$. Figure 5 presents the hierarchy of $\Omega$. As shown in the figure, all the terminal nodes are singleton for which the corresponding objective value can be obtained via (5.20). With a backward recursion, this value can then be propagated upward in the tree of supproblems. Using any ordering $\preceq_{\Omega}$ satisfying $\left(Y_{1}, Z_{1}\right) \preceq\left(Y_{2}, Z_{2}\right) \Leftrightarrow\left|Y_{1}\right| \leq$ $\left|Y_{2}\right|$, computing $\bar{F}(Y, Z)$ only depends on previously computed objective values. Table 1 presents these values for all the states. Based on the table, an optimal solution to this example is $\mathbf{y}^{*}\left(N_{3}, B_{3}\right)=(1,1,0,0)$ with $\bar{F}^{*}\left(N_{3}, B_{3}\right)=-6.4$. 


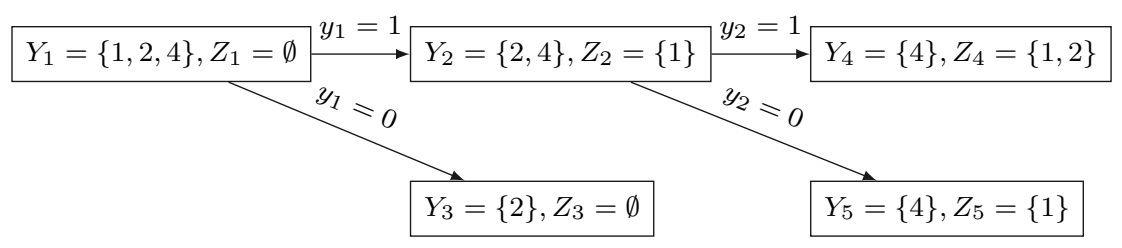

Figure 5: The state space of the example

\begin{tabular}{crrc}
\hline State Index & $R(Y, Z)$ & $F^{*}(Y, Z)$ & $\mathbf{y}^{*}(Y, Z)$ \\
\hline 5 & -12.4 & -12.4 & 1 \\
4 & 3.8 & 0 & 0 \\
3 & 0 & 0 & 0 \\
2 & 2 & -12.4 & 1 \\
1 & 6 & -6.4 & 1 \\
\hline
\end{tabular}

Table 1: The calculation details of the example

\subsubsection{A relaxation of PR}

In this section, we show that by solving a relaxation of $\mathrm{PR}$, we can solve the pricing problem more efficiently. We relax the multiplicative part of the objective (5.17) by replacing $F(Y, \varsigma, b ; \mathbf{y})$ with $F^{\prime}(Y, b ; \mathbf{y})=\sum_{j \in Y}\left(-b_{j}\right) y_{j}$. We denote this relaxation by RPR. Each instance of RPR can be converted into a special case of the maximal closure problem [29] by reversing the precedence arcs of its corresponding graph. Picard [29] shows that the maximal closure of a graph can be found by solving a maximal flow problem, and hence, can be solved very efficiently [19].

Below, we prove that solving RPR to optimality can reduce the size of PR. In order to employ the set operators, in the following, we use the set representation $C \subset N$ of the solutions to PR (recall $C_{i}^{m} \subset N$ ), rather than their vector representation y. For each $i \in C$ we have $y_{i}=1$. We also assume that $F(C) \equiv F(\mathbf{y})$.

Lemma 1. If $C_{1}, C_{2} \subseteq Y$ are two feasible solutions to $\operatorname{PR}[Y, \varsigma, b]$, then $\left(C_{1} \cup C_{2}\right) \subseteq Y$ is also a feasible solution to $\operatorname{PR}[Y, \varsigma, b]$.

Proof: A solution $C \subseteq Y$ is feasible iff for all $j \in C: B_{j} \subset C$. Moreover, we know that for any $j \in C_{1} \cup C_{2}, j$ belongs to $C_{1}$ and/or $C_{2}$. In either case, for any $i \in C_{1}$ we have $B_{j} \subset C_{1}$, and consequently, $B_{j} \subset C_{1} \cup C_{2}$. The same holds for any $j \in C_{2}$. Thus, we conclude that $\forall j \in C_{1} \cup C_{2}: B_{j} \subset C_{1} \cup C_{2}$, and consequently, $C_{1} \cup C_{2}$ is a feasible solution.

Similarly, we can prove: 
Lemma 2. If $C_{1}, C_{2} \subseteq Y$ are two feasible solutions to $\mathrm{PR}[Y, \varsigma, b]$, then $\left(C_{1} \cap C_{2}\right) \subseteq Y$ is also a feasible solution to $\operatorname{PR}[Y, \varsigma, b]$.

Using these two lemmas, we prove:

Theorem 5. If $C$ is an optimal solution to $\operatorname{RPR}[Y, b]$, then there exists an optimal solution $C^{*}$ to $\operatorname{PR}[Y, \varsigma, b]$ such that $C \subseteq C^{*}$.

Proof: We define $\bar{C}=C \backslash C^{*}$. Assuming $\bar{C} \neq \emptyset$ implies that $\exists j \in C: j \notin C^{*}$. We then have $F\left(C^{*} \cup \bar{C}\right)=\varsigma \prod_{j \in\left(C^{*} \cup \bar{C}\right)} p_{j}-\sum_{j \in\left(C^{*} \cup \bar{C}\right)} b_{j}$. Regarding the multiplicative part of the equation, since $\varsigma \geq 0$, we have $\varsigma \prod_{j \in C^{*}} p_{j} \geq \varsigma \prod_{j \in\left(C^{*} \cup \bar{C}\right)} p_{j}$. With respect to the additive part, we consider the two following cases. If $\sum_{j \in \bar{C}} b_{j}>0$, then based on Lemma 1 and the fact that $C^{*} \cup \bar{C}=C^{*} \cup C$, we have $F\left(C^{*} \cup \bar{C}\right)<F\left(C^{*}\right)$, which contradicts the optimality of $C^{*}$ in PR. If $\sum_{j \in \bar{C}} b_{j}<0$, on the other hand, then considering Lemma 2 and that $C \backslash \bar{C}=C^{*} \cap C$, we know $F^{\prime}(C \backslash \bar{C})<F^{\prime}(C)$, which contradicts the optimality of $C$ in RPR. We conclude that $\bar{C}=\emptyset$ and $C \subseteq C^{*}$. Remark that $\bar{C} \neq \emptyset$ only if $\forall j \in \bar{C}: p_{j}=1$ and $\sum_{j \in \bar{C}} b_{j}=0$, in which case $F\left(C^{*} \cup C\right)=F\left(C^{*}\right)$.

Therefore, given an optimal solution $C$ to $\operatorname{RPR}[Y, b]$ we can replace $\operatorname{PR}[Y, \varsigma, b]$ by $\operatorname{PR}[Y \backslash$ $\left.C, \varsigma \prod_{j \in C} p_{j}, b\right]$. Moreover, if $F(C)<\alpha_{i}$, we can add the corresponding column to RLF without actually solving PR explicitly. Throughout the implementation of the algorithms, we use Dinic's algorithm [16] with complexity $\mathcal{O}\left(n^{2}|E|\right)$ to solve RPR.

\subsection{Branch-and-price}

The output of the B\&P algorithm is an optimal integral solution to ILF. It starts by obtaining an optimal fractional solution to LF in the root node. Branching on the original variables $x_{i j}$ rather than the pricing variables $z_{i}^{m}$ is known to lead to more balanced search trees, and to preserve the tractability of the pricing problems [35]. Therefore, at each node, we fix a fractional variable $x_{i j}$ to either 0 or 1 . This yields two subproblems with additional constraints that can be solved by CG. The branching decisions should be imposed to PR, i.e., a variable that is fixed to zero should not be selected as entering variable in PR. To do so, we modify the precedence network, i.e., for any $\{i, j\} \in N$, fixing $x_{i j}=0$ adds $(j, i)$ and its transitive constraints to $E$. The additional constraints may empty the solution space of the restricted master problem, in which case new feasible heuristic solutions (see Section 6.2) are used to add new columns. It should be noted that the performance of a branch-and-price algorithm may improve if combined with various enhancement techniques. 
Using dual stabilization techniques can improve the convergence of CG (Wentges [40]). Moreover, cutting planes can be added in order to strengthen the relaxation of each node, and this is called branch-price-and-cut (Desrosiers and Lübbecke [15]). These techniques have not been implemented in our B\&P procedure, however.

\section{Computational results}

\subsection{Experimental setup}

All the computational experiments are performed on a Dell Latitude E7250 laptop with an Intel Core i5-5200U $(2.20 \mathrm{GHz})$ processor. A memory limit of $8 \mathrm{~GB}$ and a time limit of two hours are applied. The algorithms are coded in $\mathrm{C}++$. For solving the linear programs, we use CPLEX version 12.6.3.

We use the dataset of [39], which was generated by the random network generator RanGen [14], and which is available online ${ }^{1}$. It contains 1080 instances in 108 different settings. Each setting is defined by a probability interval PI $\in\{l, m, h\}$, an order strength value OS $\in\{0.4,0.6,0.8\}$ and $n \in\{10,20, \ldots, 120\}$. PI values represent low, medium, and high success probabilities as follows: $l$ means $p_{i} \in[0,0.2], m$ implies $p_{i} \in[0.2,0.8]$, and $h$ indicates $p_{i} \in[0.8,1]$. The success probability of each test is chosen randomly from the corresponding interval. OS is a measure of the density of a precedence network, and is defined as the number of precedence-related test pairs divided by the maximum possible number of such pairs. The cost of each test is chosen randomly from $\{0,1, \ldots, 50\}$. Each instance is identified by (PI, OS, $n, k)$, where $k \in\{1,2, \ldots, 10\}$ is the index of the instance in the setting. The three instances $(l, 0.4,90,1),(m, 0.4,90,1)$, and $(h, 0.4,90,1)$ include identical precedence networks and testing costs, but have different success probabilities.

\subsection{Implementation details}

In both DPn and DPp, we use binary search to find existing states in $\Phi$ and $\Omega$. In implementing DPp, and with minor modifications in PR, we define the states by $\left(Y, \prod_{j \in Z} p_{j}\right)$ rather than $(Y, Z)$. This alternative offers a more compact representation of $\Omega$, as any two states $(Y, Z),\left(Y, Z^{\prime}\right) \in \Omega$ with $\prod_{j \in Z} p_{j}=\prod_{j \in Z^{\prime}} p_{j}$ will map to the same objective value in $\bar{F}()$. In B\&P, we have experimented with different branching orders including the lexicographical order, the fractional variable closest to 0.5 first, the fractional variable cor-

\footnotetext{
${ }^{1}$ https://feb.kuleuven.be/public/u0004371/system_testing.htm
} 
responding to activities with most successors/predecessors first, etc. We observe that the lexicographical order performs the best.

We use a Greedy Randomized Adaptive Search Procedure (GRASP) [18] for finding highquality sets of initial columns for CG. The procedure iteratively produces heuristic sequences, and keeps track of the best produced solution. Each individual is constructed by gradually appending eligible tests to the sequence based on biased random sampling, where an eligible test with lower ratio $c_{i} /\left(1-p_{i}\right)$ has a higher selection probability.

\subsection{Comparing the results}

Table 2 compares the performance of our DP algorithm (DPn) with B\&P and the DP algorithm of Wei et al. [39] (DPk), which is the current state of the art. The comparison is performed with and without using the precedence theorems $(\mathrm{TH})$ for strengthening the networks. The column $\mathrm{TH}+\mathrm{DPn}$, for instance, shows the performance of DPn after tightening the precedence networks via the precedence theorems. Note that DPk was developed for $k: n$ systems, but in our computations we set $k=n$. The number of instances in each (OS, $n$ ) setting (30 instances) that are solved to optimality is labeled as '\#'. The average runtimes (CPU) over the solved instances are expressed in seconds. For the DP algorithms, all the unsolved instances are due to memory insufficiency, while the bottleneck of B\&P for all the unsolved instances is the time limit.

Based on the table, we observe that for all the three algorithms, the precedence theorems clearly have a strongly beneficial effect. $\mathrm{TH}+\mathrm{DPn}$ has the best performance among all the six methods. It increases the size of solvable instances by up to $100 \%$ for OS $=0.4$ when compared to the previous state of the art, DPk. In this setting, 29 out of 30 of the largest instances (with 120 tests) are solved to optimality. For OS $=0.6$, for the very first time in the literature, all the instances of the dataset are solved. The largest state space that is solved by DPn without precedence theorems corresponds to an instance with $\mathrm{OS}=0.4$ and $n=90$, which has about $3.5 \times 10^{9}$ states. This is 245 times larger than the largest state space that can be processed via $\mathrm{DPk}(\mathrm{OS}=0.4, n=60)$.

With respect to runtimes, DPn is faster than $\mathrm{DPk}$, and $\mathrm{TH}+\mathrm{DPn}$ performs better than $\mathrm{TH}+\mathrm{DPk}$. Overall, $\mathrm{TH}+\mathrm{DPn}$ outperforms $\mathrm{DPk}$ by a factor of more than 200. While both $\mathrm{DPn}$ and DPk have exponential worst-case complexity, the better performance of DPn is the result of (1) increasing the density of the precedence networks via Theorems 1, 2, and 3, (2) eliminating the explicit enumeration of UDCs in our memory management, and (3) using Theorem 4 to eliminate the unnecessary checks of the memory management technique 


\begin{tabular}{|c|c|c|c|c|c|c|c|c|c|c|c|c|c|}
\hline \multirow{2}{*}{ OS } & \multirow[b]{2}{*}{$n$} & \multicolumn{2}{|c|}{$\mathrm{TH}+\mathrm{DPn}$} & \multicolumn{2}{|c|}{$\mathrm{DPn}$} & \multicolumn{2}{|c|}{$\mathrm{TH}+\mathrm{DPk}$} & \multicolumn{2}{|c|}{$\mathrm{DPk}$} & \multicolumn{2}{|c|}{$\mathrm{TH}+\mathrm{B} \& \mathrm{P}$} & \multicolumn{2}{|c|}{$\mathrm{B} \& \mathrm{P}$} \\
\hline & & $\mathrm{CPU}$ & $\#$ & $\mathrm{CPU}$ & \# & $\mathrm{CPU}$ & \# & $\mathrm{CPU}$ & \# & $\mathrm{CPU}$ & \# & $\mathrm{CPU}$ & $\#$ \\
\hline \multirow{12}{*}{0.8} & 10 & 0.00 & 30 & 0.00 & 30 & 0.00 & 30 & 0.00 & 30 & 0.01 & 30 & 0.06 & 30 \\
\hline & 20 & 0.00 & 30 & 0.00 & 30 & 0.00 & 30 & 0.00 & 30 & 0.06 & 30 & 0.38 & 30 \\
\hline & 30 & 0.00 & 30 & 0.00 & 30 & 0.00 & 30 & 0.00 & 30 & 0.34 & 30 & 1.39 & 30 \\
\hline & 40 & 0.00 & 30 & 0.00 & 30 & 0.00 & 30 & 0.00 & 30 & 1.06 & 30 & 10.19 & 30 \\
\hline & 50 & 0.00 & 30 & 0.00 & 30 & 0.00 & 30 & 0.00 & 30 & 3.08 & 30 & 35.24 & 30 \\
\hline & 60 & 0.00 & 30 & 0.00 & 30 & 0.00 & 30 & 0.01 & 30 & 6.72 & 30 & 852.34 & 30 \\
\hline & 70 & 0.00 & 30 & 0.00 & 30 & 0.01 & 30 & 0.01 & 30 & 21.56 & 29 & - & 0 \\
\hline & 80 & 0.00 & 30 & 0.00 & 30 & 0.01 & 30 & 0.02 & 30 & 345.56 & 14 & - & 0 \\
\hline & 90 & 0.00 & 30 & 0.01 & 30 & 0.02 & 30 & 0.05 & 30 & - & 0 & - & 0 \\
\hline & 100 & 0.00 & 30 & 0.02 & 30 & 0.02 & 30 & 0.09 & 30 & - & 0 & - & 0 \\
\hline & 110 & 0.00 & 30 & 0.05 & 30 & 0.03 & 30 & 0.22 & 30 & - & 0 & - & 0 \\
\hline & 120 & 0.00 & 30 & 0.11 & 30 & 0.05 & 30 & 0.45 & 30 & - & 0 & - & 0 \\
\hline \multirow{12}{*}{0.6} & 10 & 0.00 & 30 & 0.00 & 30 & 0.00 & 30 & 0.00 & 30 & 0.02 & 30 & 0.23 & 30 \\
\hline & 20 & 0.00 & 30 & 0.00 & 30 & 0.00 & 30 & 0.00 & 30 & 0.26 & 30 & 1.59 & 30 \\
\hline & 30 & 0.00 & 30 & 0.00 & 30 & 0.00 & 30 & 0.00 & 30 & 1.27 & 30 & 226.64 & 30 \\
\hline & 40 & 0.00 & 30 & 0.00 & 30 & 0.00 & 30 & 0.01 & 30 & 18.09 & 30 & 659.79 & 24 \\
\hline & 50 & 0.00 & 30 & 0.02 & 30 & 0.00 & 30 & 0.04 & 30 & 741.22 & 30 & 6235.40 & 10 \\
\hline & 60 & 0.00 & 30 & 0.07 & 30 & 0.02 & 30 & 0.24 & 30 & 3805.90 & 21 & - & 0 \\
\hline & 70 & 0.01 & 30 & 0.31 & 30 & 0.04 & 30 & 1.12 & 30 & 4023.52 & 12 & - & 0 \\
\hline & 80 & 0.03 & 30 & 1.19 & 30 & 0.15 & 30 & 4.11 & 30 & - & 0 & - & 0 \\
\hline & 90 & 0.08 & 30 & 4.96 & 30 & 0.33 & 30 & 15.57 & 30 & - & 0 & - & 0 \\
\hline & 100 & 0.20 & 30 & 23.79 & 30 & 0.77 & 30 & 61.55 & 27 & - & 0 & - & 0 \\
\hline & 110 & 0.59 & 30 & 112.14 & 30 & 2.76 & 30 & - & 0 & - & 0 & - & 0 \\
\hline & 120 & 1.57 & 30 & 579.20 & 30 & 5.22 & 30 & - & 0 & - & 0 & - & 0 \\
\hline \multirow{12}{*}{0.4} & 10 & 0.00 & 30 & 0.00 & 30 & 0.00 & 30 & 0.00 & 30 & 0.04 & 30 & 1.49 & 30 \\
\hline & 20 & 0.00 & 30 & 0.00 & 30 & 0.00 & 30 & 0.00 & 30 & 0.69 & 30 & 42.18 & 30 \\
\hline & 30 & 0.00 & 30 & 0.01 & 30 & 0.00 & 30 & 0.03 & 30 & 79.23 & 29 & 2474.86 & 20 \\
\hline & 40 & 0.00 & 30 & 0.13 & 30 & 0.00 & 30 & 0.27 & 30 & 1099.44 & 27 & 1972.60 & 10 \\
\hline & 50 & 0.02 & 30 & 0.90 & 30 & 0.05 & 30 & 2.51 & 30 & 3770.86 & 9 & - & 0 \\
\hline & 60 & 0.08 & 30 & 11.58 & 30 & 0.19 & 30 & 31.61 & 30 & - & 0 & - & 0 \\
\hline & 70 & 0.49 & 30 & 89.33 & 30 & 0.95 & 30 & - & 0 & - & 0 & - & 0 \\
\hline & 80 & 2.05 & 30 & 877.90 & 30 & 5.10 & 30 & - & 0 & - & 0 & - & 0 \\
\hline & 90 & 12.58 & 30 & 8705.59 & 30 & 39.67 & 30 & - & 0 & - & 0 & - & 0 \\
\hline & 100 & 49.88 & 30 & - & 0 & 204.15 & 30 & - & 0 & - & 0 & - & 0 \\
\hline & 110 & 279.51 & 30 & - & 0 & 688.24 & 12 & - & 0 & - & 0 & - & 0 \\
\hline & 120 & 921.00 & 29 & - & 0 & - & 0 & - & 0 & - & 0 & - & 0 \\
\hline
\end{tabular}

Table 2: Computational results of the three algorithms

of [31]. Note that $\mathrm{TH}+\mathrm{DPk}$ outperforms $\mathrm{DPn}$, which indicates that the beneficial effect of the precedence theorems is significantly stronger that the improving effect of the new memory management technique.

The size of instances solvable by $\mathrm{B} \& \mathrm{P}$ is limited to 80 tests for $\mathrm{OS}=0.8,70$ tests for $\mathrm{OS}=0.6$, and 50 tests for $\mathrm{OS}=0.4$. More details on the performance of $\mathrm{B} \& \mathrm{P}$ are depicted in Figure 6. Further numerical details on the computational results of B\&P are presented in Table 3, where we report the total number of nodes in the B\&B tree (node) and the LP integrality gaps (\%LP). As expected, runtimes increase as $n$ increases or OS decreases. The results indicate that the quality of the LP bound is a major restriction. While the bound is tight when $\mathrm{PI}=h$, its quality deteriorates when $\mathrm{PI} \in\{l, m\}$. Consequently, the number of 

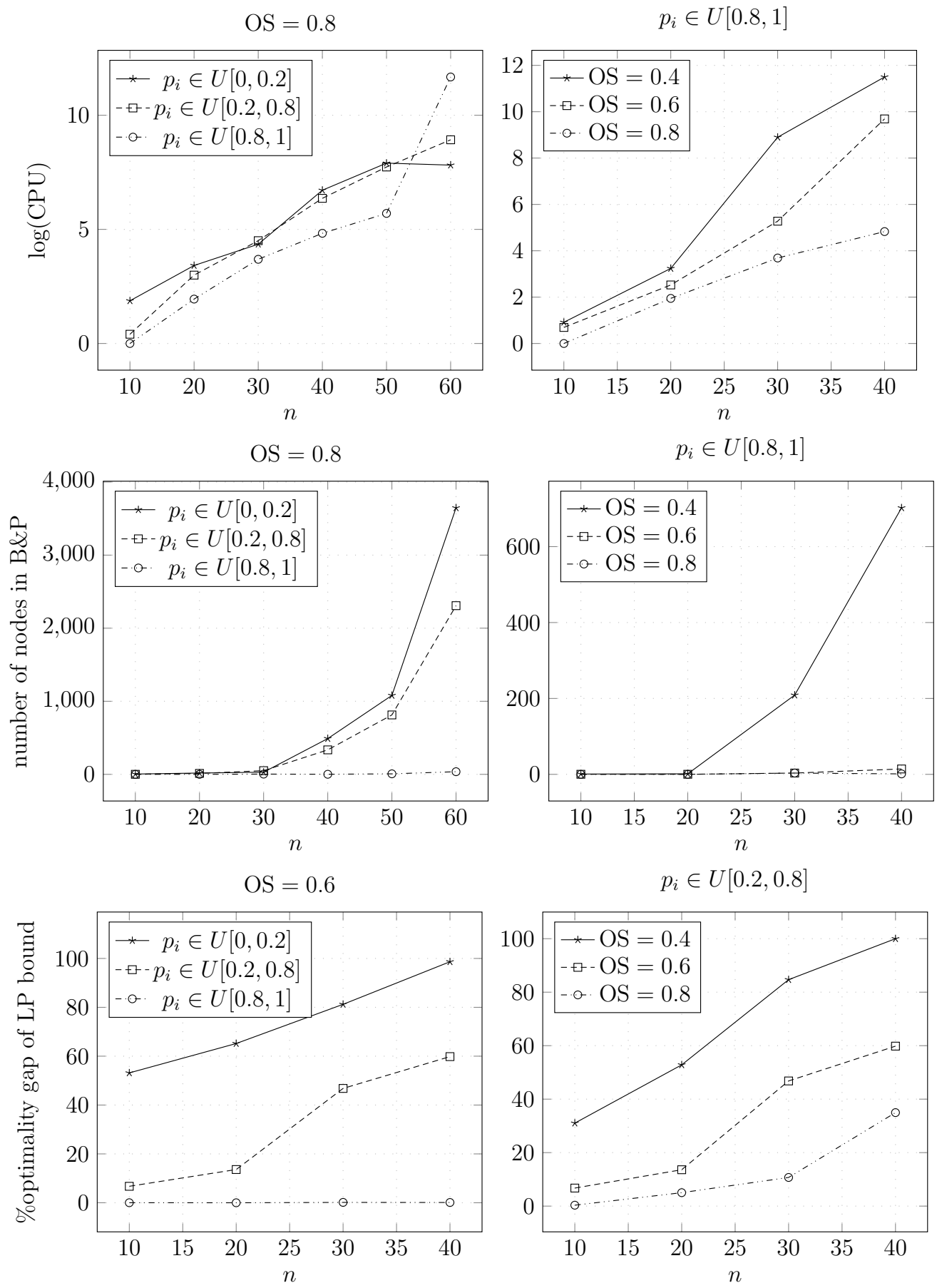

Figure 6: Computation results of $\mathrm{B} \& \mathrm{P}$ within different settings 


\begin{tabular}{|c|c|c|c|c|c|c|c|c|c|c|c|c|c|}
\hline \multirow{2}{*}{ PI } & \multirow{2}{*}{$n$} & \multicolumn{4}{|c|}{$\mathrm{OS}=0.8$} & \multicolumn{4}{|c|}{$\mathrm{OS}=0.6$} & \multicolumn{4}{|c|}{$\mathrm{OS}=0.4$} \\
\hline & & $\mathrm{CPU}$ & node & $\% \mathrm{LP}$ & $\#$ & $\mathrm{CPU}$ & node & $\% \mathrm{LP}$ & $\#$ & $\mathrm{CPU}$ & node & $\% \mathrm{LP}$ & $\#$ \\
\hline \multirow{6}{*}{$l$} & 10 & 0.13 & 4.20 & 5.23 & 10 & 0.51 & 19.00 & 53.15 & 10 & 3.12 & 155.80 & 76.57 & 10 \\
\hline & 20 & 0.61 & 18.00 & 22.37 & 10 & 2.40 & 90.80 & 65.18 & 10 & 67.85 & 2836.20 & 92.56 & 10 \\
\hline & 30 & 1.55 & 29.40 & 56.11 & 10 & 289.56 & 10305.60 & 81.22 & 10 & 3550.10 & 84750.86 & 99.89 & 7 \\
\hline & 40 & 16.50 & 489.60 & 65.78 & 10 & 691.52 & 13778.50 & 98.65 & 8 & - & - & - & 0 \\
\hline & 50 & 54.07 & 1080.00 & 84.86 & 10 & - & - & - & 0 & - & - & - & 0 \\
\hline & 60 & 49.60 & 3646.80 & 83.13 & 10 & - & - & - & 0 & - & - & - & 0 \\
\hline \multirow{6}{*}{$m$} & 10 & 0.03 & 0.60 & 0.34 & 10 & 0.15 & 6.20 & 6.74 & 10 & 1.30 & 82.60 & 31.03 & 10 \\
\hline & 20 & 0.40 & 13.00 & 5.02 & 10 & 2.10 & 84.80 & 13.60 & 10 & 58.19 & 2240.80 & 52.82 & 10 \\
\hline & 30 & 1.81 & 50.60 & 10.71 & 10 & 386.45 & 11006.40 & 46.80 & 10 & 7727.23 & 41908.00 & 84.68 & 3 \\
\hline & 40 & 11.57 & 335.00 & 34.96 & 10 & 1178.54 & 9683.67 & 59.82 & 6 & - & - & - & 0 \\
\hline & 50 & 45.68 & 813.00 & 36.62 & 10 & - & - & - & 0 & - & - & - & 0 \\
\hline & 60 & 150.41 & 2308.40 & 37.96 & 10 & - & - & - & 0 & - & - & - & 0 \\
\hline \multirow{6}{*}{$h$} & 10 & 0.02 & 0.00 & 0.00 & 10 & 0.04 & 0.20 & 0.02 & 10 & 0.05 & 0.80 & 0.05 & 10 \\
\hline & 20 & 0.14 & 0.00 & 0.00 & 10 & 0.25 & 0.20 & 0.00 & 10 & 0.51 & 1.40 & 0.08 & 10 \\
\hline & 30 & 0.80 & 3.40 & 0.03 & 10 & 3.92 & 3.40 & 0.15 & 10 & 146.48 & 208.80 & 1.37 & 10 \\
\hline & 40 & 2.50 & 1.20 & 0.10 & 10 & 323.16 & 14.20 & 0.11 & 10 & 1972.60 & 702.20 & 1.02 & 10 \\
\hline & 50 & 5.98 & 7.20 & 0.01 & 10 & 6235.40 & 402.20 & 1.10 & 10 & - & - & - & 0 \\
\hline & 60 & 2357.00 & 36.20 & 0.12 & 10 & - & - & - & 0 & - & - & - & 0 \\
\hline
\end{tabular}

Table 3: Detailed computational results for the B\&P algorithm

branched nodes increases in the latter two settings. Another observation is that the average runtime of the instances with $\mathrm{PI}=h$ and $n=60$ is higher than the average runtime of the instances with the same size but PI $\in\{l, m\}$. This implies that the branching tree is smaller when $\mathrm{PI}=h$, but obtaining an optimal fractional solution at each node is more time-consuming. By profiling our codes, we also observed that the percentage of the B\&P runtime that is spent on pricing increases as the OS decreases, the length of the probability interval increases, or the success probabilities decrease. While this percentage is consistently negligible (less than $1 \%$ ) when $\mathrm{PI}=h$ or $\mathrm{OS}=0.8$, it increases up to $30 \%$ for $\mathrm{PI}=m$ and $\mathrm{OS}=0.4$.

\section{Conclusions}

In this article, we have studied the sequential testing problem for $n: n$ systems. We have developed a linear formulation for the problem via Dantzig-Wolfe decomposition of a compact nonlinear formulation. We have proved three precedence theorems that can identify dominant precedence constraints, which are respected by at least one optimal solution. The theorems can be used in a pre-processing step to increase the density of the precedence networks.

A DP algorithm has been proposed for solving the problem. Using a novel memory management technique, our DP significantly reduces the memory requirements and the computation times. We have empirically shown that the combination of the precedence theorems and our DP is faster than the current best performing procedure by a factor of more than 
200 , and increases the size of solvable instances by up to $100 \%$.

For comparison purposes, a $\mathrm{B} \& \mathrm{P}$ algorithm has also been devised for solving the integer linear formulation. We have shown that solving a relaxation of the pricing problem can improve the efficiency of its solution method. This relaxation can be solved efficiently as a maximal flow problem. Our computational results indicate that the performance of $\mathrm{B} \& \mathrm{P}$ is inferior to the DP, however, mainly due to weak LP bounds.

\section{References}

[1] U.S. Food \& Drug Administration (FDA). http://www.fda.gov. Accessed: 2016-09-30.

[2] G. Bellala, S. K. Bhavnani, and C. Scott. Group-based active query selection for rapid diagnosis in time-critical situations. IEEE Transactions on Information Theory, 58(1): 459-478, 2012.

[3] Y. Ben-Dov. Optimal testing procedures for special structures of coherent systems. Management Science, 27(12):1410-1420, 1981.

[4] H. Boothroyd. Least-cost testing sequence. Journal of the Operational Research Society, 11(3):137-138, 1960.

[5] E. Boros and T. Ünlüyurt. Diagnosing double regular systems. Annals of Mathematics and Artificial Intelligence, 26(1-4):171-191, 1999.

[6] R. Butterworth. Some reliability fault-testing models. Operations Research, 20(2):335$343,1972$.

[7] M. F. Chang, W. Shi, and W. K. Fuchs. Optimal diagnosis procedures for k-out-of-n structures. IEEE Transactions on Computers, 39(4):559-564, 1990.

[8] S. Y. Chiu, L. A. Cox Jr, and X. Sun. Optimal sequential inspections of reliability systems subject to parallel-chain precedence constraints. Discrete Applied Mathematics, 96:327-336, 1999.

[9] K. Coolen, W. Wei, F. Talla Nobibon, and R. Leus. Scheduling modular projects on a bottleneck resource. Journal of Scheduling, 17(1):67-85, 2014.

[10] S. Creemers, R. Leus, and M. Lambrecht. Scheduling Markovian PERT networks to maximize the net present value. Operations Research Letters, 38(1):51-56, 2010. 
[11] S. Creemers, B. De Reyck, and R. Leus. Project planning with alternative technologies in uncertain environments. European Journal of Operational Research, 242(2):465-476, 2015.

[12] R. Daldal, I. Gamzu, D. Segev, and T. Ünlüyurt. Approximation algorithms for sequential batch-testing of series systems. Naval Research Logistics (NRL), 63(4):275-286, 2016.

[13] B. De Reyck and R. Leus. R\&D-project scheduling when activities may fail. IIE Transactions, 40:367-384, 2008.

[14] E. Demeulemeester, M. Vanhoucke, and W. Herroelen. RanGen: A random network generator for activity-on-the-node networks. Journal of Scheduling, 6(1):17-38, 2003.

[15] J. Desrosiers and M. E. Lübbecke. Branch-price-and-cut algorithms. Encyclopedia of Operations Research and Management Science. John Wiley 85 Sons, Chichester, pages 109-131, 2011.

[16] E. A. Dinic. Algorithm for solution of a problem of maximum flow in a network with power estimation. Soviet Mathematics Doklady, 11(5):1277-1280, 1970.

[17] S. O. Duffuaa and A. Raouf. An optimal sequence in multicharacteristics inspection. Journal of Optimization Theory and Applications, 67(1):79-86, 1990.

[18] T. A. Feo and M. G. Resende. Greedy randomized adaptive search procedures. Journal of Global Optimization, 6(2):109-133, 1995.

[19] L. R. Ford and D. R. Fulkerson. Flows in Networks. Princeton University Press, 1962.

[20] M. R. Garey. Optimal task sequencing with precedence constraints. Discrete Mathematics, 4(1):37-56, 1973.

[21] B. Gluss. An optimum policy for detecting a fault in a complex system. Operations Research, 7(4):468-477, 1959.

[22] S. M. Johnson. Optimal sequential testing. Technical Report RM-1652, Rand Corporation, 1956.

[23] J. Kanet. New precedence theorems for one-machine weighted tardiness. Mathematics of Operations Research, 32(3):579-588, 2007. 
[24] F. P. Kelly. A remark on search and sequencing problems. Mathematics of Operations Research, 7(1):154-157, 1982.

[25] D. Madigan, S. Mittal, and F. Roberts. Efficient sequential decision-making algorithms for container inspection operations. Naval Research Logistics (NRL), 58(7):637-654, 2011.

[26] R. K. Martin. Large Scale Linear and Integer Optimization: A Unified Approach. Springer Science \& Business Media, 2012.

[27] L. G. Mitten. An analytic solution to the least cost testing sequence problem. Journal of Industrial Engineering, 11(1):17, 1960.

[28] C. L. Monma and J. B. Sidney. Sequencing with series-parallel precedence constraints. Mathematics of Operations Research, 4(3):215-224, 1979.

[29] J.-C. Picard. Maximal closure of a graph and applications to combinatorial problems. Management Science, 22(11):1268-1272, 1976.

[30] C. N. Potts. An algorithm for the single machine sequencing problem with precedence constraints. In Combinatorial Optimization II, pages 78-87. Springer, 1980.

[31] S. Rostami, S. Creemers, and R. Leus. Precedence theorems and dynamic programming for the single-machine weighted tardiness problem. Technical Report KBI_1715, KU Leuven, Faculty of Economics and Business, 2017.

[32] S. N. Salloum. Optimal testing algorithms for symmetric coherent systems. PhD thesis, University of Southern California, 1979.

[33] S. N. Salloum and M. A. Breuer. Fast optimal diagnosis procedures for k-out-of-n: G systems. IEEE Transactions on Reliability, 46(2):283-290, 1997.

[34] N. Samphaiboon and Y. Yamada. Heuristic and exact algorithms for the precedenceconstrained knapsack problem. Journal of Optimization Theory and Applications, 105 (3):659-676, 2000.

[35] M. Savelsbergh. A branch-and-price algorithm for the generalized assignment problem. Operations Research, 45(6):831-841, 1997.

[36] T. Ünlüyurt. Sequential testing of complex systems: A review. Discrete Applied Mathematics, 142:189-205, 2004. 
[37] F. Vanderbeck. On Dantzig-Wolfe decomposition in integer programming and ways to perform branching in a branch-and-price algorithm. Operations Research, 48(1):111$128,2000$.

[38] B. J. Wagner and D. J. Davis. Discrete sequential search with group activities. Decision Sciences, 32:557-573, 2001.

[39] W. Wei, K. Coolen, and R. Leus. Sequential testing policies for complex systems under precedence constraints. Expert Systems with Applications, 40(2):611-620, 2013.

[40] P. Wentges. Weighted dantzig-wolfe decomposition for linear mixed-integer programming. International Transactions in Operational Research, 4(2):151-162, 1997. 